\title{
ON CAUCHY'S FUNCTIONAL EQUATION
}

\author{
WOLFGANG B. JURKAT
}

0 . Introduction. In this note we shall solve two open problems ${ }^{1}$ concerning Cauchy's functional equation

$$
f(x+y)=f(x)+f(y)
$$

for real-valued functions of a real variable.

P. Erdös [2] asked, after learning about a preliminary result of S. Hartman [3], whether one obtains all functions satisfying $(C)$ for almost all pairs $(x, y)$ by simply redefining the functions satisfying (C) for all $(x, y)$ in an arbitrary manner on sets of measure zero. This turns out to be the case (Theorem I), in particular, if $f$ satisfies (C) for almost all $(x, y)$ and is also measurable or only bounded from below on a set of positive measure, then $f(x)=c x$ holds almost everywhere as a consequence of well-known theorems of A. Ostrowski [5], H. Kestel$\operatorname{man}[4]$.

I. Halperin asked whether (C) for all $(x, y)$ in conjunction with

$$
f\left(\frac{1}{x}\right)=\frac{1}{x^{2}} f(x) \quad(\text { all } x \neq 0)
$$

already ensures $f(x)=x f(1)$. This also turns out to be true (Theorem II).

1. Theorem I. Let $f(x)$ be real valued, defined for almost all real $x$, and suppose that $(\mathrm{C})$ holds for almost all pairs $(x, y)$ in the sense of plane measure (Lebesgue). Then there exists a real-valued function $F(x)$, defined for all real $x$ and satisfying $(\mathrm{C})$ for all pairs $(x, y)$, which coincides with $f(x)$ for almost all $x$ in the sense of linear measure (Lebesgue). These requirements determine $F(x)$ uniquely.

Proof. By Fubini's theorem there are null-sets $N$ and $N_{x}$ such that (C) holds if $x \notin N, y \notin N_{x}$. Denote the complement of $N$ by $M$ and notice, in particular, that $f$ must be defined on $M$. First we show that (C) holds whenever $x \in M, y \in M, x+y \in M$. Fix $x, y$ for the moment and pick $z$ such that $z \notin N_{x+y}, y+z \notin N_{x}, z \notin N_{y}$. This is possible by avoiding three null-sets for $z$. But then we have

Presented to the Society, December 23, 1963; received by the editors December 23, 1963.

${ }^{1}$ These were taken from a list of open problems on Functional Equations given by J. Aczel at the Syracuse University Mathematics Colloquium on December 6, 1963. 


$$
\begin{aligned}
f(x+y+z) & =f(x+y)+f(z), \\
f(x+y+z) & =f(x)+f(y+z), \\
f(y+z) & =f(y)+f(z),
\end{aligned}
$$

and the result follows.

Next we show

$$
\begin{aligned}
f\left(x_{1}\right)+f\left(y_{1}\right)=f\left(x_{2}\right)+f\left(y_{2}\right) \quad \text { if } x_{1}+y_{1}=x_{2}+y_{2}, & \\
x_{1} & \in M, y_{1} \in M, x_{2} \in M, y_{2} \in M,
\end{aligned}
$$

by picking $z \in M$ such that $y_{1}^{\prime}=y_{1}-z \in M, y_{2}^{\prime}=y_{2}-z \in M, x_{1}+y_{1}^{\prime}$ $=x_{2}+y_{2}^{\prime}=x_{1}+y_{1}-z=x_{2}+y_{2}-z \in M$. This is possible by avoiding four null-sets for $z$ and implies

$$
\begin{aligned}
f\left(y_{1}\right) & =f\left(y_{1}^{\prime}\right)+f(z), & f\left(y_{2}\right) & =f\left(y_{2}^{\prime}\right)+f(z), \\
f\left(x_{1}+y_{1}^{\prime}\right) & =f\left(x_{1}\right)+f\left(y_{1}^{\prime}\right), & f\left(x_{2}+y_{2}^{\prime}\right) & =f\left(x_{2}\right)+f\left(y_{2}^{\prime}\right)
\end{aligned}
$$

by what we already know. Hence

$$
f\left(x_{1}\right)+f\left(y_{1}\right)=f\left(x_{1}+y_{1}^{\prime}\right)+f(z)=f\left(x_{2}+y_{2}^{\prime}\right)+f(z)=f\left(x_{2}\right)+f\left(y_{2}\right) .
$$

Finally we show that given $x_{1} \in M, x_{2} \in M, x_{3} \in M$ there exist $y_{1} \in M, y_{2} \in M$ such that

(2) $x_{1}+x_{2}+x_{3}=y_{1}+y_{2}$ and $f\left(x_{1}\right)+f\left(x_{2}\right)+f\left(x_{3}\right)=f\left(y_{1}\right)+f\left(y_{2}\right)$.

This is done by picking $z \in M$ such that $z^{\prime}=x_{3}-z \in M, y_{1}=x_{1}+z \in M$, $y_{2}=x_{2}+z^{\prime}=x_{2}+x_{3}-z \in M$ (avoid four null-sets) and then combining the consequences

$$
f\left(x_{3}\right)=f(z)+f\left(z^{\prime}\right), \quad f\left(y_{1}\right)=f\left(x_{1}\right)+f(z), \quad f\left(y_{2}\right)=f\left(x_{2}\right)+f\left(z^{\prime}\right) .
$$

In order to define $F$ we notice that every real number $z$ is of the form $x+y$ with $x \in M, y \in M$ (simply pick $x \in M$ such that $y=z-x$ $\in M)$. Define $F(z)$ to be $f(x)+f(y)$, which is single-valued because of (1). For $z \in M$ the equation (C) implies $F(z)=f(z)$. Now take two arbitrary real numbers $z_{1}, z_{2}$ of the forms $x_{1}+y_{1}, x_{1} \in M, y_{1} \in M$ and $x_{2}+y_{2}, x_{2} \in M, y_{2} \in M$, respectively. By applying (2) twice we obtain two numbers $z_{1}^{\prime} \in M, z_{2}^{\prime} \in M$ such that

$$
\begin{aligned}
x_{1}+y_{1}+x_{2}+y_{2} & =z_{1}^{\prime}+z_{2}^{\prime} \quad \text { and } \\
f\left(x_{1}\right)+f\left(y_{1}\right)+f\left(x_{2}\right)+f\left(y_{2}\right) & =f\left(z_{1}^{\prime}\right)+f\left(z_{2}^{\prime}\right) .
\end{aligned}
$$

But the left side in the last equation equals $F\left(z_{1}\right)+F\left(z_{2}\right)$ while the right side equals $F\left(z_{1}^{\prime}+z_{2}^{\prime}\right)=F\left(z_{1}+z_{2}\right)$, thus proving (C) for $F$ with unrestricted variables.

It remains to show the uniqueness of $F$. So let us take two func- 
tions $F_{1}(x)$ and $F_{2}(x)$ satisfying (C) for all pairs $(x, y)$ and coinciding on a set $M$ which includes almost all $x$. The difference $F=F_{1}-F_{2}$ also satisfies (C) and vanishes on $M$; but every real number $z$ is of the form $x+y, x \in M, y \in M$ showing that $F(z)=F(x)+F(y)=0$ holds generally.

2. Remarks. If we combine Theorem I with sufficient conditions for $F(x)$ to equal $c x$ we obtain the results mentioned in the introduction. But these special consequences could also be obtained more directly by using the fact that the sum-set $C=A+B=\{z \mid z=x+y$, $x \in A, y \in B\}$ always contains an open interval if $A$ and $B$ are of positive measure (e.g., use the continuity of the convolution of the corresponding characteristic functions restricted to a large enough interval). This is essentially the idea in [4], but although very simple under the conditions prevailing there, the proof remains rather involved here. If, however, $f$ is measurable, the discussion in $[1$, p. 143] suggests integrating the functional equation resulting for $g_{\lambda}(x)$ $=e^{i \lambda f(x)}$, where we choose for $\lambda$ two real values with irrational quotient. This turns out to be a very simple procedure.

It is obvious that in the proof of Theorem I only a few specific properties of real numbers have been used. Consequently there will be analogues for very general domains and ranges of $f$. More important, the concept of measure enters only via null-sets. Once Fubini's theorem has been used, only the following properties are important. The family of null-sets is closed under the application of linear mappings and finite unions, but does not include the whole space as an element. Notice that sets of the first category, for instance, satisfy the same properties. Cf. [3].

3. Theorem II. Let $f(x)$ be real valued, defined for all real $x$, and suppose that $(\mathrm{C})$ holds for all $(x, y)$ together with $\left(\mathrm{C}^{\prime}\right)$. Then $f(x)=x f(1)$ holds for all $x$.

Proof. Obviously $f(0)=0, f(-x)=-f(x)$. For $x \neq 0,1$ we have in turn

$$
\begin{aligned}
\frac{1}{x(x-1)} & =\frac{1}{x-1}-\frac{1}{x} \\
\frac{1}{x^{2}(x-1)^{2}} f\left(x^{2}-x\right) & =\frac{1}{(x-1)^{2}} f(x-1)-\frac{1}{x^{2}} f(x), \\
f\left(x^{2}\right)-f(x) & =x^{2}\{f(x)-f(1)\}-(x-1)^{2} f(x), \\
f\left(x^{2}\right) & =2 x f(x)-x^{2} f(1) .
\end{aligned}
$$


The last equation is also true for $x=0$ or $x=1$. Now use $4 x y=(x+y)^{2}$ $-(x-y)^{2}$ to obtain

$$
\begin{aligned}
f(4 x y)= & 2(x+y) f(x+y)-(x+y)^{2} f(1) \\
& -\left\{2(x-y) f(x-y)-(x-y)^{2} f(1)\right\} \\
= & 4 x f(y)+4 y f(x)-4 x y f(1) .
\end{aligned}
$$

Hence

$$
f(x y)=x f(y)+y f(x)-x y f(1),
$$

in particular, for $y=1 / x(x \neq 0)$,

$$
\begin{aligned}
& f(1)=x f\left(\frac{1}{x}\right)+\frac{1}{x} f(x)-f(1)=\frac{2}{x} f(x)-f(1), \\
& f(x)=x f(1) .
\end{aligned}
$$

The last equation holds for $x=0$ as well.

Here again it is possible to generalize the range and domain of $f$.

Added in proof. J. Aczél held a similar lecture on Some unsolved problems in the theory of functional equations at Oberwolfach in October, 1963, which appeared in Arch. Math. 15 (1964), 435-444 (cf. Problems 13 and 14). The problem of Erdös was solved independently by N. G. de Bruijn in a manuscript On almost additive functions which he sent to me in September, 1964. The problem of Halperin was solved independently by $\mathrm{S}$. Kurepa in a manuscript on The Cauchy functional equation and scalar product in vector spaces, Glasnik Mat.-Fiz. Astronom. Društvo Mat. Fiz. Hrvatske Ser. II (to appear), which he sent to me in March, 1964. Halperin's problem was also solved independently by S. L. Segal in a letter sent to me in March, 1964.

\section{REFERENCES}

1. J. Aczél, Vorlesungen über Funktionalgleichungen und ihre Anwendungen, Birkhäuser Verlag, Basel-Stuttgart, 1961.

2. P. Erdös, Problem 310, Colloq. Math. 7 (1960), 311.

3. S. Hartman, A remark about Cauchy's equation, Colloq. Math 8 (1961), 77-79.

4. H. Kestelman, On the functional equation $f(x+y)=f(x)+f(y)$, Fund. Math. 34 (1947), 144-147.

5. A. Ostrowski, Mathematische Miszellen. XIV. Über die Funktionalgleichung der Exponentialfunktion und verwandte Funktionalgleichungen, Jber. Deutsch. Math.Verein. 38 (1929), 54-62.

SYRACUSE UNIVERSITY 